\title{
Basilea Il: los países en desarrollo y la diversificación de la cartera
}

\author{
Stephany Griffith-Jones, Miguel Ángel Segoviano \\ y Stephen Spratt
}

$\mathrm{L}$

nueva propuesta para el acuerdo de Basilea tiene por objeto armonizar de una mejor manera los requisitos de capitalización con el riesgo real que los bancos tomen. Aquí se sostiene que las nuevas propuestas podrían aumentar significativa e inadecuadamente el costo o reducir el volumen de los créditos bancarios que se otorgan a los países en desarrollo, debido a que harían mucho más estrictos los requisitos necesarios para proporcionarles capital. El hecho de que las propuestas

Stephany Griffith-Jones

Professorial Fellow,

Instituto de Estudios del Desarrollo,

Universidad de Sussex

Consultora de la CEPAL

○.griffith-jones@ids.ac.uk

Miguel Angel Segoviano

Candidato al doctorado en

London School of Economics

- m.a.segoviano@ise.ac.uk

Stephen Spratt

Vicepresidente,

Intelligence Capital

- s.spratt@gam.com no hayan tenido en cuenta las ventajas de la diversificación internacional implica que se está sobreestimando el riesgo a nivel de la cartera. En el presente trabajo se demuestra que, para una serie de variables (como la rentabilidad bancaria) y para una serie de períodos, el grado de correlación entre las economías desarrolladas es mayor que el que se registra entre países desarrollados y en desarrollo. También se demuestra, mediante el uso de simulaciones, que el riesgo para una cartera distribuida entre economías desarrolladas y en desarrollo es menor que en el caso de una cartera concentrada exclusivamente en economías desarrolladas. En consecuencia, instamos al Comité de Basilea a introducir explícitamente las evidentes ventajas de la diversificación internacional. 


\section{I}

\section{Introducción}

La primera vez que expresamos nuestras reservas con respecto a las consecuencias que podría acarrear el propuesto Nuevo Acuerdo de Capital de Basilea, conocido como Basilea II, fue tras la publicación por el Comité de Basilea del Segundo Documento Consultivo (CP2) en enero de 2001. ${ }^{1}$ A partir de entonces, se han introducido modificaciones a las propuestas que, en cierta medida, responden a nuestras reservas originales.

El último trabajo que preparamos sobre el tema fue publicado en el Financial Regulator en septiembre de 2002. ${ }^{2}$ En él reiteramos nuestras dudas acerca de las consecuencias que podrían tener las propuestas para las economías en desarrollo, evaluamos los posibles efectos de las modificaciones que se habían anunciado hasta esa fecha y pusimos de relieve dos aspectos que aún resultaban preocupantes y que se exponen a continuación.

El primero de estos aspectos es que la aplicación generalizada del enfoque basado en calificaciones internas del riesgo ${ }^{3}$ por los bancos que operan a nivel internacional se traduciría en un aumento (disminución) significativo de los requisitos de capital para otorgar créditos a los deudores peor (mejor) clasificados. En la medida en que los requisitos de capital influyan en el precio y en la disponibilidad de crédito bancario internacional, habría un acentuado incremento del costo o una reducción del monto de los préstamos internacionales a las economías en desarrollo y emergentes. Dado que el nivel actual de estos préstamos es muy bajo, existe el peligro de que tal situación se "institucionalice", de manera que incluso si mejorara la situación mundial, disminuirían notablemente las posibilidades de que los créditos de la banca internacional contribuyeran al desarrollo de los países más pobres.

$\square$ Deseamos agradecer a Danielle Nouy, Karsten Von Kleist, Marian Micu, Serge Jeanneau y Philipp Klingelhofer por proporcionarnos valiosos datos y estímulo para esta investigación. También agradecemos a los profesores Charles Goodhart y Avinash Persaud por sus sabios consejos en los aspectos conceptuales y prácticos del trabajo. Por cierto, cualquier error corre por nuestra cuenta.

${ }^{1}$ Véase Griffith-Jones y Spratt (2001).

2 Véase Griffith-Jones, Spratt y Segoviano (2002).

${ }^{3}$ El enfoque (o método o fórmula) basado en calificaciones internas del riesgo corresponde a lo que en inglés se denomina internal ratings based approach (IRB approach).
Durante mucho tiempo se ha sostenido que una de las principales ventajas de invertir en las economías en desarrollo y emergentes radica en que su correlación con los mercados "maduros" es relativamente baja. Como se demuestra más adelante, este hecho es evidente $\mathrm{y}$, en consecuencia, son obvios los beneficios que - en función del total de la cartera- podrían obtener los bancos con carteras internacionales bien diversificadas. Es decir, un banco cuya cartera de crédito esté bien distribuida en una gama de mercados relativamente no correlacionados, se expone a menos problemas simultáneos en todos esos mercados que un banco cuyos créditos se concentran en un número más reducido de mercados relativamente más correlacionados. Así, pues, para armonizar correctamente los requisitos de capital con los riesgos reales que asume el banco, el Acuerdo debiera tener en cuenta este efecto de la cartera: los requisitos de capital para un banco con una cartera de créditos internacionales bien diversificada debieran reflejar el hecho de que su riesgo total es menor que el de bancos con una cartera más concentrada. Actualmente las propuestas no contienen consideración alguna de esta índole, lo que indicaría que, al menos en este ámbito, los requisitos de capital pueden no reflejar correctamente el riesgo real.

El argumento de que la correlación de los activos es variable resulta evidente. Además, al parecer se reafirma la idea de que esta variabilidad influye en el nivel total de riesgo de una cartera $y$, en consecuencia, debe manifestarse en los requisitos de capital. En efecto, el Comité de Basilea de Supervisión Bancaria ha reconocido este hecho con las modificaciones que ya ha introducido respecto a los préstamos para pymes. Tras la publicación del documento consultivo en enero del 2001, hubo una preocupación generalizada por el impacto negativo que podría tener en las pymes un fuerte aumento de los requisitos de capital para acceder a préstamos. Después de intensos cabildeos, el Comité reconsideró el tema y convino en que debía separarse el trato a las pymes de los demás préstamos a empresas, y que los deudores cuyas ventas anuales sumaran menos de 50 millones de euros debían recibir una reducción promedio de los requisitos de capital de un $10 \%$ respecto de las empresas grandes. El fundamento de esta modificación es que la posibilidad 
de que una gran cantidad de pymes se declaren simultáneamente en incumplimiento de obligaciones es menor que la posibilidad de que lo haga un pequeño grupo de grandes deudores. Es decir, la correlación en la probabilidad del incumplimiento es inferior. En consecuencia, con una cartera de préstamos bien diversificada en una amplia gama de pymes, los riesgos en función de la cartera total serán menores que con una cartera concentrada en unos pocos deudores grandes.

Los resultados de nuestro trabajo empírico sugieren que se justifica introducir una modificación similar con respecto a la diversificación internacional.

El segundo aspecto preocupante es que la utilización de indicadores de riesgo sensibles a las fluctuaciones de los mercados - como lo prevé el enfoque basado en las calificaciones internas- es intrínsecamente procíclica. El hecho de que los requisitos de capital marchen a la par con el ciclo económico implica una ampliación de dicho ciclo a medida que los créditos cambian de categoría de riesgo según mejora o empeora la situación económica. En consecuencia, la tendencia natural de los operadores del mercado - incluidos los banqueros - a subestimar los riesgos en períodos de bonanza y a sobrestimarlos en épocas de recesión se institucionalizará y legitimará mediante la reglamentación propuesta. Por lo tanto, al experimentarse un repunte de la economía, la impresión general de que el riesgo es menor se traduciría en una flexibilización de las condiciones crediticias, lo que fortalecería aún más esta impresión de bajo riesgo pero tal vez llevaría a prolongar el período de bonanza y favorecer la acumulación de niveles más altos de riesgo sistémico. A la inversa, durante una fase descendente o una recesión, el aumento de los requisitos de capital, según lo establece el enfoque basado en las calificaciones internas, reduciría el incentivo para otorgar créditos y - junto con la dificultad de conseguir capital en un período de recesión- podría ocasionar una restricción pronunciada del crédito, en cuyo caso ni siquiera los proyectos potencialmente rentables podrían conseguir financiamiento. El peligro radica en que un cambio desfavorable de la situación económica pueda derivar en una recesión, o que una recesión existente se prolongue o intensifique.

Las preocupaciones que hemos expuesto sobre los efectos adversos que podría acarrear Basilea II se examinaron en el marco de un análisis más general. De acuerdo con éste, se sostuvo que los principales problemas que enfrentan los países en desarrollo para tener acceso al financiamiento externo con miras a promover su crecimiento y desarrollo son: i) el bajo nivel actual de flujos financieros de todo tipo (en especial, pero no exclusivamente, de préstamos bancarios) y ii) la creciente tendencia cortoplacista y procíclica de dichos flujos (Griffith-Jones, 2002). Dada nuestra visión de este panorama general desalentador, continúa preocupándonos seriamente el hecho de que las propuestas de Basilea II puedan exacerbar estas tendencias negativas, en lugar de contrarrestarlas.

En este artículo daremos a conocer los resultados del trabajo empírico que realizamos para abordar el primero de los dos aspectos preocupantes anteriormente señalados. En nuestro más reciente estudio al respecto se sugiere que una de las razones por las cuales los requisitos de capital contemplados en las nuevas propuestas podrían ser inadecuadamente altos para las economías en desarrollo y emergentes es el hecho de que no toman en cuenta las ventajas de la diversificación internacional. Además se plantea que, si pudiera demostrarse que la correlación de los préstamos de países desarrollados a países desarrollados es superior a la que se da cuando se trata de préstamos a países desarrollados y en desarrollo, podría sostenerse que el nivel de riesgo de una cartera de préstamos diversificada internacionalmente entre deudores de países tanto desarrollados como en desarrollo, sería menor - en función del total de la cartera- que si se concentrara solamente en el otorgamiento de créditos a países desarrollados. De ser así, sería posible, y ciertamente conveniente, que el Comité de Basilea incorporara las ventajas de la diversificación internacional en el nuevo Acuerdo.

Este argumento es similar al utilizado para respaldar las últimas modificaciones, efectuadas en noviembre de 2001, las que, respecto de los préstamos otorgados a las empresas, se tradujeron en un aplanamiento de la curva que determina los requerimientos de capitalización como una función de las probabilidades de incumplimiento de los créditos (IRB curve). En las propuestas de enero de 2001, se partía implícitamente de la base de que la correlación media de los activos era igual a 0,2 . Sin embargo, tras investigaciones empíricas llevadas a cabo por el Comité de Basilea, se propuso modificar la fórmula basada en las calificaciones internas, de manera que el coeficiente de correlación disminuyera de 0,2 a 0,1 a medida que aumentara la probabilidad de incumplimiento. Es decir, mientras mayor fuese la probabilidad de incumplimiento de las empresas, menor sería la correlación, puesto que la quiebra o la insolvencia pueden obedecer a diversos factores no sistémicos que no siempre influyen en las perspectivas de las demás empresas.

La afirmación de que la correlación de los activos es variable tiene fundamentos evidentes. Además, 
parece sólida la sugerencia de que esta variabilidad influye en el nivel total de riesgo de una cartera y, en consecuencia, debe manifestarse en los requisitos de capital. Por lo tanto, aplicamos este criterio en nuestro trabajo empírico que, como se indicará más adelante, justifica plenamente las razones para introducir una modificación similar en la fórmula basada en las calificaciones internas de los créditos diversificados internacionalmente.

Durante largo tiempo se ha sostenido que una de las principales ventajas de invertir en las economías en desarrollo radica en que su correlación con los mercados maduros es relativamente baja. Por lo tanto, nuestra primera hipótesis podría plantearse de la siguiente manera:

Hipótesis 1: El grado de correlación entre los sectores real y financiero de las economías desarrolladas es superior al que existe entre las economías desarrolladas y en desarrollo.

Sometimos a prueba esta hipótesis, primero, centrándonos concretamente en la relación directa entre los préstamos y la rentabilidad de la banca internacional $\mathrm{y}$, en seguida, de una manera más general y a modo de respaldo. Todos los resultados que obtuvimos apoyaron de manera significativa la validez de este planteamiento. Esto proporcionó la base para una segunda hipótesis, relacionada específicamente con la labor que lleva a cabo el Comité de Basilea.

Hipótesis 2: La relación riesgo/rendimiento de una cartera de préstamos diversificada que incluye tanto regiones desarrolladas como regiones emergentes y en desarrollo es más eficiente que la de una cartera concentrada exclusivamente en países desarrollados. En consecuencia, el nivel general de riesgo de la primera, medido por las pérdidas no esperadas, deberá ser menor.

Para poner a prueba esta hipótesis, simulamos el nivel de pérdidas no esperadas de dos carteras, una de créditos que se distribuyen parejamente entre regiones desarrolladas y en desarrollo, y otra de créditos que se otorgan sólo a regiones desarrolladas. Los resultados de estas simulaciones respaldan inequívocamente la segunda de nuestra hipótesis, pues demuestra que las pérdidas no esperadas que debería enfrentar en un caso extremo una cartera concentrada puramente en deudores de países desarrollados serían alrededor de $25 \%$ más altas que las de una cartera repartida entre países desarrollados y en desarrollo.

A nuestro juicio, todas las pruebas que realizamos, utilizando diversas variables en diversos períodos, proporcionan sólida evidencia en apoyo a nuestra hipótesis. Esa evidencia se fortalece aún más con los resultados de nuestras simulaciones de carteras de créditos, las que mediante la aplicación de un método análogo al que utiliza la mayoría de los bancos con sistemas de análisis de riesgos técnicamente más avanzados, demuestran las ventajas de la diversificación internacional, tal como las concebirían los grandes bancos. En su conjunto, la evidencia indica que, para no castigar injustamente a las economías en desarrollo y emergentes, el Comité de Basilea debería examinar detenidamente la conveniencia de incorporar las ventajas de la diversificación internacional en el documento consultivo final, próximo a publicarse. Confiamos en que las pruebas que presentaremos a continuación demuestren la validez de este punto de vista.

El presente artículo consta de cinco secciones. Tras la presente introducción (sección I), se describen las fuentes de información y la metodología utilizada (sección II), se presentan los resultados del trabajo econométrico (sección III), se incluyen las simulaciones de dos carteras de crédito (sección IV) y se analizan las connotaciones de los resultados obtenidos, formulando algunas conclusiones (sección V). En los apéndices A y B, por último, se presentan algunos detalles técnicos sobre el trabajo estadístico y de simulación.

\section{II}

\section{Fuentes e información}

Los países considerados son los siguientes:

- Países en desarrollo: Argentina, Brasil, Chile, Ecuador, México, Panamá, Perú y Venezuela; Filipinas, Indonesia, Malasia, Tailandia y la República de Corea; Bulgaria, Polonia y Rusia; Nigeria y Sudáfrica.
- Países desarrollados: Canadá y Estados Unidos; Japón; Alemania, España, Francia, Italia y el Reino Unido.

- Otros: Singapur; Finlandia, Grecia, Irlanda y Portugal.

Las variables analizadas aparecen en el cuadro 1. 
CUADRO 1

Clasificación, descripción y otras características de las variables analizadas, diversos períodos

\begin{tabular}{|c|c|c|c|c|c|}
\hline Clasificación & Clave & Descripción & Período & Frecuencia & Fuente \\
\hline Sector financiero & ROA & Rentabilidad de los activos (bancarios) & $1988-2001$ & Anual & The Banker \\
\hline Sector financiero & ROC & $\begin{array}{l}\text { Rentabilidad del capital de primera línea } \\
\text { (bancario) }\end{array}$ & $1988-2001$ & Anual & The Banker \\
\hline Sector financiero & Sindicados & Márgenes de los préstamos sindicados & $1993-2002$ & Mensual & $\begin{array}{l}\text { Banco de Pagos } \\
\text { Internacionales (BPI) }\end{array}$ \\
\hline Bonos & $\mathrm{GBI}^{\mathrm{a}}$ & Índice global de los bonos & $1987-2002$ & Diaria & JP Morgan/Reuters \\
\hline Bonos & $\mathrm{EMBI}^{\mathrm{b}}$ & Índice de los bonos de mercados emergentes & $1987-2002$ & Diaria & JP Morgan/Reuters \\
\hline Bonos & $\mathrm{EMBI}+{ }^{\mathrm{c}}$ & $\begin{array}{l}\text { Índice de los bonos de mercados emergentes } \\
\text { Plus }\end{array}$ & $1987-2002$ & Diaria & JP Morgan/Reuters \\
\hline Acciones & IFC $G^{d}$ & $\begin{array}{l}\text { Standard\&Poor y Corporación Financiera } \\
\text { Internacional (CFI) (mundial) }\end{array}$ & $1990-2002$ & Diaria & $\mathrm{CFI} / \mathrm{S} \& \mathrm{P}$ \\
\hline Acciones & IFC $\mathrm{I}^{\mathrm{e}}$ & S\&P y CFI (invertible) & 1990-2002 & Diaria & $\mathrm{CFI} / \mathrm{S} \& \mathrm{P}$ \\
\hline Acciones & COMP & $\begin{array}{l}\text { Países en desarrollo enumerados: índice } \\
\text { compuesto de acciones }\end{array}$ & 1990-2002 & Diaria & Reuters \\
\hline Macro & GDP & Tasa de crecimiento del PIB & $1985-2000$ & Semestral & $\begin{array}{l}\text { FMI, Banco Mundial } \\
\text { (cálculos de los autores) }\end{array}$ \\
\hline Macro & GDP HP & $\begin{array}{l}\text { Descomposición del PIB según } \\
\text { Hodrick-Prescott }\end{array}$ & $1950-1998$ & Anual & $\begin{array}{l}\text { Estadísticas nacionales } \\
\text { (cálculos de los autores) }\end{array}$ \\
\hline Macro & STIR & Tasa de interés nominal de corto plazo & $1985-2000$ & Semestral & $\begin{array}{l}\text { Estadísticas nacionales (BPI) } \\
\text { o FMI, CFI }\end{array}$ \\
\hline Macro & STIRR & Tasa de interés real de corto plazo & $1985-2000$ & Semestral & $\begin{array}{l}\text { Estadísticas nacionales (BPI) } \\
\text { o FMI, CFI }\end{array}$ \\
\hline
\end{tabular}

Fuente: Elaboración propia.

a El GBI se compone de bonos públicos internos de tasa fija que se transan regularmente. El mercado de deuda interna de los países comprendidos es líquido y de libre acceso para los inversionistas extranjeros. El GBI excluye los pagarés con interés variable, los bonos perpetuos, los bonos con vencimiento a menos de un año, los bonos destinados a mercados internos por razones tributarias y los bonos con opción de compra, de venta y convertibles.

b El EMBI es un índice de bonos de mercados emergentes, elaborado por J.P. Morgan. Incluye los bonos Brady, denominados en dólares, los eurobonos, y los préstamos e instrumentos de deuda interna emitidos por entidades soberanas y cuasi soberanas.

c El EMBI+ es una ampliación del EMBI. Registra a todos los instrumentos de deuda denominados en moneda extranjera de los mercados emergentes.

d El IFC G (mundial) es un índice accionario de los mercados emergentes elaborado conjuntamente por la Corporación Financiera Internacional (CFI) y Standard \& Poor (S\&P). No toma en cuenta las restricciones a la propiedad extranjera que limitan el acceso a algunos mercados y acciones determinadas.

e El IFC I (invertible) se ajusta de manera de indicar las restricciones a las inversiones extranjeras en los mercados emergentes. En consecuencia, representa un panorama más preciso del universo real al alcance del inversionista.

\section{III}

\section{Resultados}

Todas las pruebas de significación estadística realizadas respaldan plenamente nuestra primera hipótesis. En cada uno de los casos se realizaron pruebas en las que se hizo uso de la función de distribución acumulativa, a fin de determinar la validez de los resultados obtenidos.
Las pruebas tuvieron por objeto demostrar, para cualquier nivel dado de correlación, la probabilidad de que en la serie que considera los créditos de países desarrollados a países en desarrollo la correlación sea menor que en la serie que presenta los créditos de países 
desarrollados a otros países desarrollados. En los gráficos 1 y 2 se muestran los resultados obtenidos en dos de estas pruebas, como una ilustración de que en todos los casos la correlación desarrollados/desarrollados predomina por sobre la correlación desarrollados/ en desarrollo (los demás resultados figuran en el apéndice $\mathrm{A})$.

Es decir, para cualquier nivel de correlación $x$, la probabilidad de que la correlación efectiva sea menor

GRAFICO 1
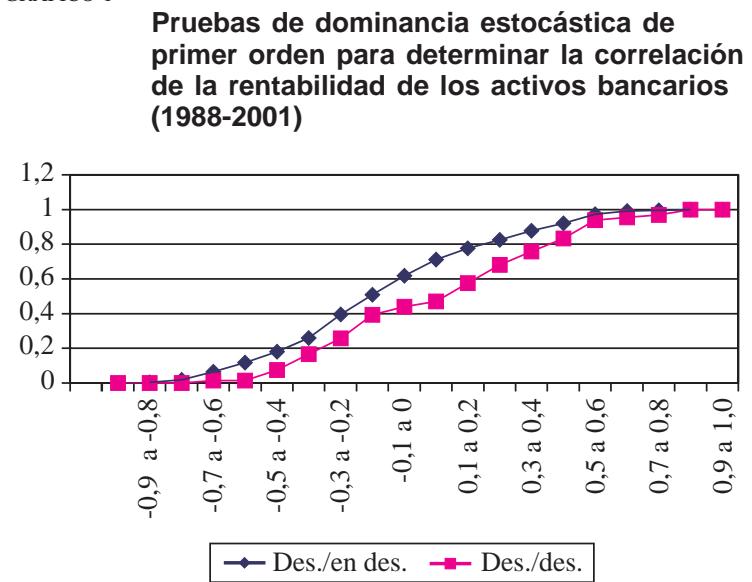

Fuente: Análisis hecho para este estudio. que $x$ es mayor en la serie de países desarrollados/en desarrollo que en la serie de países desarrollados/desarrollados.

Los resultados que aparecen en el cuadro 2 refuerzan la primera de nuestras hipótesis, tanto en un sentido general como específico. A continuación presentamos los resultados específicos del sector financiero y, luego, aquellos relativos a las variables económicas y financieras de carácter más general.
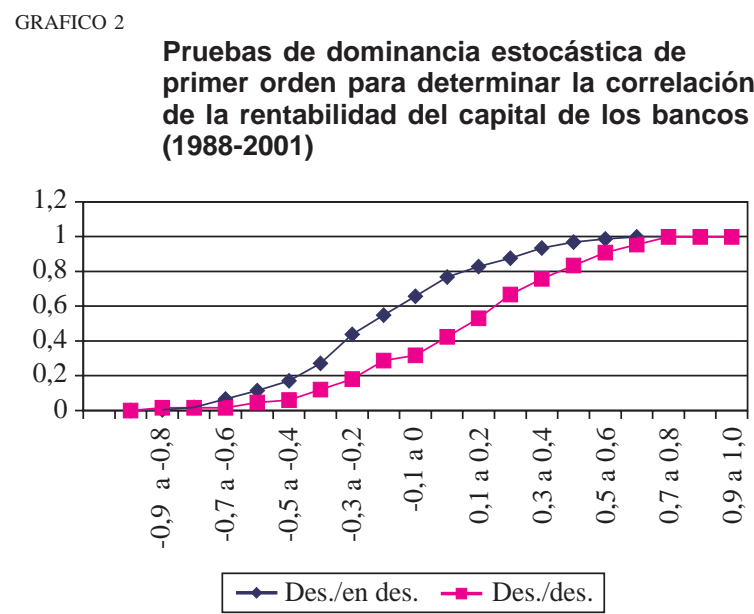

Fuente: Análisis hecho para este estudio.

CUADRO 2

Coeficiente de correlación media

\begin{tabular}{|c|c|c|c|c|c|}
\hline Variable & Período & Frecuencia & $\begin{array}{l}\text { Coeficiente de } \\
\text { correlación media } \\
\text { entre países } \\
\text { desarrollados }\end{array}$ & $\begin{array}{c}\text { Coeficiente de } \\
\text { correlación media } \\
\text { entre países desarrollados } \\
\text { y en desarrollo }\end{array}$ & $\begin{array}{l}\text { Prueba estadística } \\
(\mathrm{HO}: \mathrm{Mx}=\mathrm{My})^{\mathrm{a}}\end{array}$ \\
\hline Sindicados & 1993-2002 & Mensual & 0,37 & 0,14 & $3,33(3,29)$ \\
\hline ROA & 1988-2001 & Anual & 0,10 & $-0,08$ & $4,40(3,29)$ \\
\hline ROC & 1988-2001 & Anual & 0,14 & $-0,11$ & $6,92(3,29)$ \\
\hline PIB & $1985-2000$ & Semestral & 0,44 & 0,02 & $9,08(3,29)$ \\
\hline PIB HP & 1950-1998 & Anual & 0,35 & 0,02 & $9,41(3,29)$ \\
\hline $\mathrm{STIR}^{\mathrm{b}}$ & $1985-2000$ & Semestral & 0,72 & 0,23 & $11,09(3,29)$ \\
\hline STIRR $^{\mathrm{c}}$ & $1985-2000$ & Semestral & 0,66 & 0,22 & $10,93(3,29)$ \\
\hline GBI-EMBI & 1991-2002 & Diario & 0,78 & 0,53 & $5,45(3,29)$ \\
\hline GBI-EMBI & 1991-1997 & Diario & 0,90 & 0,74 & $4,64(3,29)$ \\
\hline GBI-EMBI & 1998-2002 & Diario & 0,42 & 0,09 & $5,87(3,29)$ \\
\hline CFI-COMP & $1990-2000$ & Diario & 0,58 & $-0,15$ & $7,83(3,29)$ \\
\hline IFCG-COMP & $1990-2000$ & Diario & 0,58 & $-0,17$ & $8,06(3,29)$ \\
\hline
\end{tabular}

Fuente: Análisis hecho para este estudio.

a Valor crítico de $0,05 \%$ de la prueba unilateral (One-tailed test).

b Tasa de interés nominal de corto plazo.

c Tasa de interés real de corto plazo. 
Como puede apreciarse en el cuadro 2, se pusieron a prueba todos los resultados a fin de asegurarse de que fueran estadísticamente significativos. En todos los casos lo fueron, con un nivel de confiabilidad del $99,5 \%$. Además, se rechazó claramente la hipótesis nula de que, en promedio, las correlaciones medias de ambas series fuesen iguales $\left(\mathrm{H}_{0}: \mathrm{Mx}=\mathrm{My}\right)$.

\section{Análisis}

En el cuadro 1 se observa claramente que en nuestras pruebas utilizamos una amplia gama de variables financieras, de mercado y macroeconómicas. Si bien cabría sugerir que podría criticarse individualmente cada una de estas variables, por constituir un indicador en cierto modo imperfecto del comportamiento de un mercado financiero, argumentaríamos con fuerza que las distorsiones en los datos probablemente se contrarrestarían recíprocamente, pues es poco probable que obedezcan a causas comunes. En consecuencia, el hecho de que todas las pruebas estadísticas realizadas, sea cual fuere la variable, el período considerado o la frecuencia apunten en la misma dirección, y que luego de ser sometidas a otras pruebas resulten estadísticamente significativas, demuestra inequívocamente la validez de nuestra primera hipótesis.

En el caso de los márgenes de los préstamos bancarios sindicados, y partiendo del supuesto razonable de que son indicativos del nivel de riesgo de tales préstamos $-\mathrm{y}$, en consecuencia, una variable sustitutiva de la probabilidad de incumplimiento-, es evidente que los riesgos, medidos de esta forma, han tenido una tendencia a aumentar y disminuir conjuntamente dentro de las regiones desarrolladas que ha sido mayor que la registrada en regiones desarrolladas y en desarrollo en conjunto. Por consiguiente, este primer resultado parece respaldar nuestra hipótesis. Es decir, en el período 1993-2002, un banco con una cartera de préstamos distribuida entre las principales regiones desarrolladas y en desarrollo habría gozado de los beneficios de la diversificación a nivel de la cartera. En efecto, la correlación entre los riesgos que acompañan a los créditos otorgados a cada una de estas regiones habría sido menor que si la cartera hubiese estado concentrada únicamente en mercados desarrollados.

Asimismo, el hecho de que la correlación entre la rentabilidad de los bancos en mercados en desarrollo y la de los bancos en mercados desarrollados sea levemente negativa, en tanto que la correlación de la rentabilidad de los bancos dentro de mercados desarrollados es ligeramente positiva, refuerza aún más nuestra hipótesis sobre las ventajas de la diversificación. No obstante, si bien hay muchos factores que influyen en la rentabilidad del sistema bancario de un país, parece razonable suponer que uno de los más importantes sería la incidencia de los préstamos impagos en su economía. En general, es probable que la solidez y rentabilidad consiguiente de la economía de un país influya notablemente en la rentabilidad de su sector bancario. De esta manera, en el período considerado, un banco que hubiese otorgado créditos a bancos y empresas de diversos países desarrollados y en desarrollo se habría visto favorecido por la diversificación, en comparación con uno que hubiese concentrado sus préstamos exclusivamente en mercados desarrollados.

Asimismo, los resultados obtenidos del análisis de las variables macroeconómicas, pese a ser más generales, indican que la tendencia de las economías desarrolladas a evolucionar paralelamente es mayor que la de economías desarrolladas y en desarrollo consideradas en conjunto. Si se parte de la base de que la incidencia de los créditos impagos de una economía es, al menos en forma parcial, inversamente proporcional a la tasa de crecimiento del producto interno bruto (PIB), entonces habría menos probabilidades de que en los bancos cuya cartera esté diversificada a nivel internacional se produzca simultáneamente un incremento pronunciado de la incidencia de esta clase de préstamos. En cambio, en el caso de los bancos que concentren sus operaciones de crédito en mercados maduros, cuya correlación es mayor, la probabilidad sería más alta. Similares consecuencias pueden esperarse si se consideran las fluctuaciones de las tasas de interés de corto plazo como un indicador aproximado del ciclo económico - ya que el alza de las tasas indica el término del ciclo ascendente y la baja de las tasas el fin del ciclo descendente-, lo que respalda aún más nuestra tesis. Tal como sucede con el crecimiento del PIB, el hecho de que en los países desarrollados los ciclos económicos - y en consecuencia las fluctuaciones de las tasas de interés de corto plazo- estén más correlacionados que en una combinación de países desarrollados y en desarrollo, indica que seguramente la incidencia de préstamos impagos será mayor en los primeros que en la segunda.

Para muchos operadores del mercado, las fluctuaciones del precio y el rendimiento de los bonos gubernamentales representan un buen indicador de las variables macroeconómicas fundamentales de un país y de las opiniones de los mercados sobre las perspectivas económicas de éste. El hecho de que el valor de 
los bonos de países desarrollados marche mucho más a la par que el de bonos de una combinación de países desarrollados y en desarrollo indica que en los primeros hay más correlación entre esas variables macroeconómicas y la percepción de los mercados al respecto. Similares resultados se obtuvieron al analizar mercados bursátiles de algunos países. Nuestras pruebas revelan una menor correlación entre los mercados bursátiles de los mercados desarrollados y en desarrollo. En consecuencia, en la medida en que el mercado bursátil de un país sea el reflejo de dichas variables macroeconómicas y de la percepción de los inversionistas acerca del país, el hecho de que haya menos correlación cuando se trata de países desarro- llados y en desarrollo confirma aún más nuestra primera hipótesis.

Las pruebas mencionadas respaldan claramente nuestra hipótesis de que el riesgo general de un banco cuya cartera esté distribuida a nivel internacional entre deudores de países desarrollados y en desarrollo será menor que el de aquél que otorgue créditos exclusivamente a países desarrollados. Con el objeto de comprobar esta hipótesis en el contexto específico de una cartera de préstamos de un banco, realizamos un ejercicio de simulación, a fin de evaluar las pérdidas no esperadas que podrían producirse en una cartera diversificada entre países desarrollados, y otra diversificada entre regiones desarrolladas y en desarrollo.

\section{IV}

\section{Simulaciones de carteras de préstamos}

Para comprobar nuestra segunda hipótesis, construimos dos carteras de préstamos simuladas a fin de evaluar las pérdidas no esperadas que cada una de ellas podría sufrir. De esta manera, se puede comparar directamente el comportamiento simulado de una cartera diversificada entre regiones desarrolladas y en desarrollo con el de una cuyos préstamos se concentren exclusivamente en los mercados desarrollados.

A continuación presentamos en detalle el contexto básico para nuestra metodología y los resultados obtenidos. En el apéndice B se presenta información más detallada, así como datos técnicos sobre la construcción de las carteras simuladas.

\section{Contexto}

La calidad crediticia de la cartera de un banco puede variar en cualquier momento, de manera que se hace necesario calcular con frecuencia las pérdidas que la institución podría sufrir en diversas circunstancias. Dada la variabilidad a que está sujeta una cartera, es probable que el cálculo de las reservas preventivas no sea el mismo para los distintos períodos. La diferencia entre las reservas preventivas calculadas para los distintos períodos (debido a cambios en la calidad de los créditos) hace que el banco pueda sufrir pérdidas, las que en situaciones extremas podrían mermar su capital. Estas pérdidas se denominan "pérdidas no esperadas". De hecho, nuestra segunda hipótesis postula que las pérdidas no esperadas de una cartera diversificada entre mercados desarrollados y en desarrollo serán menores que las de una concentrada exclusivamente en mercados desarrollados. En principio, respaldan esta hipótesis los resultados obtenidos a partir de nuestro análisis estadístico, en virtud del cual se comprobó que el coeficiente de correlación de las carteras que combinan mercados de países desarrollados y en desarrollo es menor que el de las que se concentran exclusivamente en mercados desarrollados.

\section{Simulación}

La metodología utilizada corresponde a una modificación del conocido modelo CreditMetrics, que ha sido aplicado ampliamente para calcular las pérdidas no esperadas de una cartera. Adoptando un método análogo, construimos dos carteras simuladas: en la primera, los préstamos se distribuyeron parejamente en las principales regiones desarrolladas y en desarrollo; en la segunda, éstos se concentraron exclusivamente en las regiones desarrolladas. Posteriormente, programamos un algoritmo, en el cual se simularon 10.000 "escenarios de calidad" diferentes que podrían afectar a dichas carteras y provocar, en consecuencia, cambios en la clasificación crediticia. ${ }^{4}$ Cada escenario muestra

\footnotetext{
${ }^{4}$ Entre las regiones en desarrollo figuran: África y el Medio Oriente, Asia y el Pacífico, los países en desarrollo de Europa y América Latina. Entre las regiones desarrolladas se incluyen: los países de la Unión Europea no pertenecientes a la Unión Económica y Monetaria, aquellos pertenecientes a dicha unión monetaria, otros países industrializados y centros extraterritoriales.
} 
un cambio en el valor de mercado de los activos de los acreedores de la cartera y, por lo tanto, permite evaluar la diferencia entre la calidad inicial y la calidad final del crédito. Una vez simulados los escenarios de calidad de la cartera de préstamos, se pueden calcular las pérdidas o ganancias generadas por la diferencia entre su calidad inicial y final.

Las pérdidas o ganancias registradas en este proceso de simulación se utilizaron para construir un histograma, en el que se resume la distribución de las pérdidas de la cartera. A partir de esta distribución, se definió un valor en riesgo (VaR) a partir del cual se obtiene el valor de las pérdidas no esperadas de la cartera. ${ }^{5}$ Estas pérdidas, divididas por el monto total de la cartera, representan el porcentaje que, dada una probabilidad determinada (definida en función del percentil elegido), podría perderse en un caso extremo.

\section{Resultados}

Los resultados de nuestras simulaciones (cuadro 3) respaldan firmemente nuestra segunda hipótesis. Como puede apreciarse en el cuadro, las pérdidas no esperadas de la cartera concentrada en deudores de países desarrollados son, en promedio, casi un $23 \%$ más elevadas que si la cartera hubiese estado distribuida entre países desarrollados y en desarrollo.

\section{Análisis}

Las simulaciones muestran claramente que, a nivel de carteras, la relación entre los riesgos y el rendimiento de los bancos es más favorable cuando los créditos están distribuidos a nivel internacional. Dado que los requisitos de capital supuestamente deberían cubrir las pérdidas no esperadas, el hecho de que en nuestra simulación dichas pérdidas sean menores cuando la cartera está diversificada revela que, para indicar con precisión el riesgo real que pueden enfrentar los bancos, Basilea II debería tener en cuenta el efecto de la diversificación.

Naturalmente, siempre es posible poner en tela de juicio las premisas sobre las cuales se basa una simulación. En el presente trabajo, intentamos asegurar que nuestros postulados fueran lo más razonables posible. Uno de los aspectos que consideramos detenidamente fue que, al partir de la base de que dentro de los países no había diversificación por industrias, se podría impedir que los países desarrollados, que generalmente tienen más industrias que los países en desarrollo, se vieran favorecidos por la diversificación. Sin embargo, llegamos a la conclusión de que posiblemente se haya sobrestimado las presuntas ventajas de esta diversificación. Algunos estudios empíricos recientes, elaborados por el Comité de Basilea de Supervisión Bancaria, confirman esta impresión (Acharya, Hasan y Saunders, 2002). Utilizando datos correspondientes a 105 bancos italianos para el período 1993-1999, dichos autores buscaron pruebas empíricas que respaldaran las ventajas que, en teoría, tendría la diversificación industrial, sectorial y geográfica. Aunque sus resultados son hasta cierto punto sorprendentes, parecen reforzar tanto las premisas sobre las cuales se basa la simulación de la cartera de préstamos (es decir, que

CUADRO 3

Comparación de carteras no diversificadas por industrias

(Pesos y porcentajes)

\begin{tabular}{|c|c|c|c|c|c|c|}
\hline \multirow[b]{2}{*}{ Percentil } & \multicolumn{2}{|c|}{$\begin{array}{l}\text { Cartera diversificada entre países desarrollados } \\
\text { y en desarrollo } \\
\text { Total de préstamos }=117625333,00\end{array}$} & \multicolumn{3}{|c|}{$\begin{array}{l}\text { Cartera diversificada entre países desarrollados } \\
\frac{\text { Total de préstamos }=117625333,00}{}\end{array}$} & \multirow[b]{2}{*}{$\begin{array}{l}\text { Diferencia } \\
\text { porcentua }\end{array}$} \\
\hline & $\begin{array}{l}\text { Valor de las } \\
\text { pérdidas }\end{array}$ & $\begin{array}{c}\text { Pérdidas } \\
\text { no esperadas }\end{array}$ & Percentil & $\begin{array}{l}\text { Valor de las } \\
\text { pérdidas }\end{array}$ & $\begin{array}{c}\text { Pérdidas } \\
\text { no esperadas }\end{array}$ & \\
\hline 99,8 & 22595312 & 19,21 & 99,8 & 27869349 & 23,69 & $+23,34$ \\
\hline 99,9 & 26390246 & 22,44 & 99,9 & 32187075 & 27,36 & $+21,96$ \\
\hline
\end{tabular}

Fuente: Análisis hecho para este estudio.

\footnotetext{
${ }^{5}$ Naturalmente, la aplicación del modelo de cálculo del valor en riesgo a la gestión del riesgo plantea muchos problemas y es objeto de críticas. Véase, por ejemplo, Zigrand y Danielsson (2001) y Persaud (2001). Sin embargo, este tipo de problemas escapa al alcance del presente trabajo. En nuestro estudio, la simulación tiene
}

por objeto mostrar, en términos generales, la diferencia relativa en materia de pérdidas no esperadas que podría experimentar cada cartera, como hacen actualmente muchos grandes bancos que operan a nivel internacional. 
Comparación de dos carteras simuladas, diversificadas por industrias

(Pesos y porcentajes)

\begin{tabular}{|c|c|c|c|c|c|c|}
\hline \multirow[b]{2}{*}{ Percentil } & \multicolumn{2}{|c|}{$\begin{array}{c}\text { Cartera diversificada entre países desarrollados } \\
\text { y en desarrollo } \\
\text { Total de préstamos }=117625333,00\end{array}$} & \multicolumn{4}{|c|}{ Cartera diversificada entre países desarrollados } \\
\hline & $\begin{array}{l}\text { Valor de las } \\
\text { pérdidas }\end{array}$ & $\begin{array}{c}\text { Pérdidas } \\
\text { no esperadas }\end{array}$ & Percentil & $\begin{array}{l}\text { Valor de las } \\
\text { pérdidas }\end{array}$ & $\begin{array}{c}\text { Pérdidas } \\
\text { no esperadas }\end{array}$ & $\begin{array}{l}\text { Diferencia } \\
\text { porcentual }\end{array}$ \\
\hline 99,8 & 15111321 & 12,85 & 99,8 & 17665318 & 15,02 & 16,90 \\
\hline 99,9 & 15358788 & 13,06 & 99,9 & 17960850 & 15,27 & 16,94 \\
\hline
\end{tabular}

Fuente: Análisis hecho para este estudio.

no hay efectos de diversificación aparentes entre las industrias) como, lo que es aún más importante, las conclusiones generales de nuestro trabajo empírico.

Por consiguiente, a partir de los resultados obtenidos con respecto a la rentabilidad y el riesgo de los créditos bancarios, concluimos que, en el caso de los bancos (italianos) examinados en el estudio, la mayor diversificación de los préstamos entre industrias produce una relación riesgo/rendimiento ineficiente, y la diversificación por sectores produce una relación riesgo/rendimiento ineficiente para los bancos cuyo nivel de riesgo es relativamente elevado. Por otra parte, la diversificación geográfica efectivamente mejora la relación riesgo/rendimiento de los bancos cuyo nivel de riesgo es bajo o moderado (Acharya, Hasan y Saunders, 2002, p. 5).

Sin embargo, para cerciorarnos de que esta hipótesis no distorsiona los resultados de la simulación, realizamos una segunda serie de simulaciones. En este caso, se partió de la base de que había diversificación, tanto geográfica como por industrias. Como se observa en el cuadro 4, esta modificación, que asemeja más la simulación a la realidad, tiene como consecuencia la reducción del $50 \%$ de las pérdidas no esperadas de la cartera; por lo tanto, ahora se acercan más al $8 \%$, cifra que se registra frecuentemente en la práctica y que constituye la base de los requisitos de capital que, a juicio del Comité de Basilea, se aplican al sistema en su conjunto.

Asimismo, esta modificación también reduce, aunque en menor medida, la diferencia entre las pérdidas no esperadas simuladas en ambas carteras. Sin embargo, la diferencia, de casi $17 \%$ en promedio, continúa siendo muy significativa $\mathrm{y}$, por lo tanto, reafirma aún más la solidez de los resultados obtenidos.

Otro aspecto que examinamos fue el hecho de que las correlaciones no son constantes en el tiempo. Por supuesto, el peligro radica en que en los mercados emergentes, las correlaciones aumentan radicalmente en situaciones de crisis, a medida que el contagio propaga la crisis de un país o región a otro. En este caso, puede ocurrir que una cartera diversificada en varias regiones emergentes y en desarrollo se vea afectada simultáneamente en cada una de ellas. Sin embargo, aunque ésta sea probablemente la impresión general acerca del comportamiento de los mercados emergentes en situaciones de crisis, quizás sólo sea aplicable a un número limitado de casos en que se den determinados requisitos. Actualmente, y de hecho en la mayoría de los casos, ello no es así. Kaminsky, Reinhart y Vegh (2002) examinaron 200 años de crisis financieras en países desarrollados y en desarrollo en busca de pruebas de contagio, y llegaron a la conclusión de que es posible que se produzca un contagio "virulento" como el aquí descrito, pero únicamente en ciertas circunstancias. Entre las principales crisis que han afectado a los mercados de países emergentes desde 1980, se sostiene que la crisis mexicana de 1982, la devaluación mexicana de 1994, la devaluación del baht tailandés de 1997 y la crisis rusa de 1998 de hecho tuvieron un efecto de contagio importante. No obstante, salvo en el caso de la moratoria rusa, que afectó a todas las regiones emergentes y en desarrollo y en medida sorprendente al mundo desarrollado (Davis, 1999), el contagio resultante de otras crisis se limitó a la región de origen. Por lo tanto, si la cartera hubiese estado distribuida en todas las regiones emergentes y en desarrollo, no se habrían producido simultáneamente problemas de la magnitud descrita. Por otra parte, en casos más recientes — como la devaluación en Brasil en 1999, en Turquía a comienzos de 2001 y los problemas desencadenados en Argentina hacia fines de 2001 - el contagio ha sido mucho menor y no se ha convertido en un fenómeno de alcance general en los mercados emergentes. 
En Kaminsky, Reinhart y Vegh (2002) se sugiere que, para que una crisis se propague más allá de los límites regionales, debe ser precedida por un período de auge (o "burbuja") de inversiones. De esta manera, los actores que se encuentran fuera de la región se involucran en lo que sucede en ella, por lo que en cierta medida la crisis puede propagarse a otras regiones emergentes e incluso a regiones en desarrollo por medio de acreedores comunes. Por cierto, el contexto actual no está marcado por un período de bonanza en materia de flujos de capital hacia las economías emergentes y en desarrollo. Además, es poco probable que se produzca nuevamente este tipo de situaciones en el futuro previsible, si se garantiza que no se darán las condiciones necesarias para contagiar a todo el siste-

\section{V}

\section{Conclusiones}

El objetivo expreso del propuesto nuevo Acuerdo de Capital de Basilea es el de armonizar mejor los requisitos de capitalización con el riesgo real que los bancos tomen. Se sostiene que este proceso hará que los créditos bancarios tengan un marco regulatorio más sólido y eliminará las numerosas anomalías que se perciben en el Acuerdo vigente. En el presente trabajo, hemos tratado de demostrar que las nuevas propuestas podrían aumentar el costo y reducir el volumen de los créditos bancarios que se otorgan a los países en desarrollo, debido a que harían mucho más estrictos los requisitos para proporcionar capital a deudores de alto riesgo. Ello se justifica debido a que el capital asociado con los deudores peor (mejor) clasificados debe aumentar (disminuir) significativamente según las circunstancias, lo que simplemente reflejaría una mayor precisión en la medición del riesgo.

Sin embargo, como se demostró en este artículo, el hecho de que hasta ahora las propuestas no hayan tenido en cuenta las ventajas de la diversificación internacional indica que, al menos en este caso, no se ha medido el riesgo con exactitud. Es decir, al excluir la posibilidad de que los requisitos de capital impuestos a los bancos excluyan los efectos de la diversificación, se impone una medida inexacta del riesgo real a nivel de la cartera. Actualmente, los bancos más tecnificados suelen tomar en cuenta los efectos de la diversificación en sus estrategias de préstamos internacionales. El hecho de que la propuesta de Basilea II no permita ma. Así, las ventajas de una diversificación amplia seguirán existiendo.

Kaminsky y Reinhart (2002) también destacan este hecho. Según sus investigaciones, las perturbaciones financieras que se producen en la "periferia" (los países en desarrollo) sólo tienen repercusiones sistémicas más allá de la región vecina del país afectado cuando atañen a los mercados de activos de alguno de los centros financieros más importantes (el mundo desarrollado). Según dichos autores: los centros financieros cumplen así una función decisiva en la propagación de las perturbaciones financieras; cuando ellos se mantienen a salvo, los problemas que enfrenta un mercado emergente se detienen en el límite de la región (ibid., p. 3).

tomar en cuenta dicho efecto sugiere que el capital regulatorio que se asociará a los préstamos otorgados a los países en desarrollo será superior al que los bancos se reservarían - y de hecho se reservan- basándose en sus propios modelos.

El Comité de Basilea ya ha introducido una serie de cambios a las propuestas presentadas en enero de 2001, entre los cuales los más importantes son los que modifican la fórmula de calificación interna del riesgo para tener en cuenta la variable de correlación de los activos en relación con el incumplimiento, y los cambios relativos a las pymes. Cuando se dio a conocer el segundo documento consultivo, la impresión general fue que los préstamos a las pymes podrían verse afectados si aumentaban las exigencias de capitalización para otorgarlos. Tras intensas negociaciones, el Comité de Basilea ha reconsiderado el asunto. No cabe duda de que los cambios generales que se han introducido en la fórmula de calificación interna del riesgo en el crédito a las empresas (en el cual la curva se ha aplanado significativamente), evidentemente favorecerán a las pymes. El Comité ha ido más allá y en julio de 2002 dio a conocer un documento que destacaba los aspectos principales en los que se había llegado a acuerdo, entre los cuales estaba el de dar a las pymes el siguiente tratamiento:

Reconociendo los diferentes riesgos asociados con los créditos a las pymes, y de acuerdo con el enfoque 
basado en la calificación interna del riesgo de los créditos otorgados a empresas, los bancos podrán hacer una distinción entre los préstamos a pymes (es decir, a empresas cuyas ventas anuales sean inferiores a 50 millones de euros) y los préstamos a empresas más grandes. De acuerdo con el tratamiento propuesto, se permitirá que los requisitos de capital para la participación en inversiones de las pymes sean menores que aquellos exigidos a las empresas más grandes. La reducción del monto de capital requerido podrá ser incluso de 20\%, según la importancia del prestatario, y debería traducirse en una baja de aproximadamente $10 \%$, en promedio, para el conjunto de las pymes prestatarias en el marco de la calificación interna del riesgo de los préstamos otorgados al sector empresarial (Comité de Basilea de Supervisión Bancaria, 2002).

Así, en el caso de los créditos otorgados a pymes, el Comité de Basilea ha reconocido el impacto que puede tener la diferencia de correlación de los activos en el nivel de riesgo de la cartera. Los resultados obtenidos justifican plenamente que se introduzca una modificación similar respecto de los créditos diversificados a nivel internacional.

Evidentemente, corresponde al propio Comité de Basilea determinar la forma en que habrán de incorporarse estas conclusiones. Conociendo su experiencia y capacidad profesional, a estas alturas no quisiéramos sugerir cómo podrían realizarse tales modificaciones. Sin embargo, dados los cambios que ya se han introducido en la calificación interna del riesgo para los créditos a las pymes, así como el hecho de que las modificaciones que proponemos parecerían tener una base al menos igualmente sólida, no existen razones teóricas, empíricas ni prácticas que impidan realizar cambios encaminados a incorporar las ventajas de la diversificación internacional.

APENDICE A

\section{Pruebas de la función de distribución acumulada ${ }^{\mathrm{a}}$}

A.1. Pruebas de predominio estocástico de primer orden para determinar las correlaciones de los márgenes de los préstamos de consorcio (1993-2002)

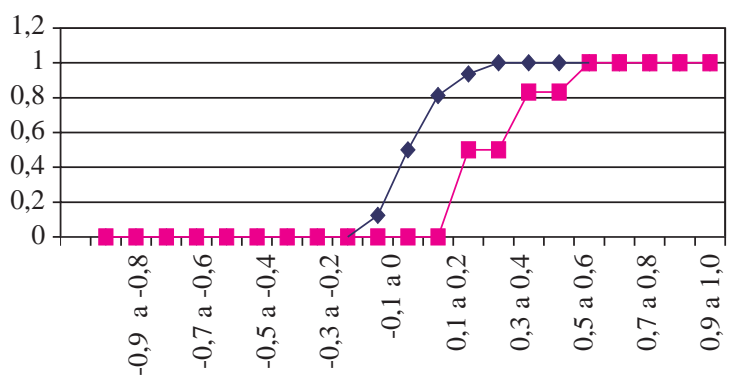

A.3. Pruebas de dominancia estocástica de primer orden para determinar la correlación de la rentabilidad de los activos bancarios (1988-2001)

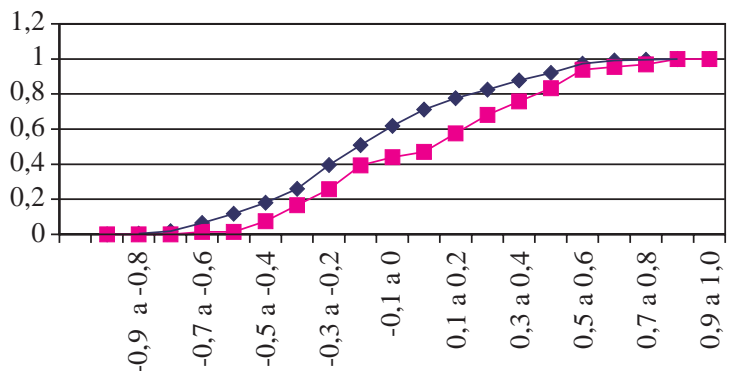

A.2. Pruebas de dominancia estocástica de primer orden para determinar las correlaciones de la rentabilidad del capital de los bancos (1988-2001)

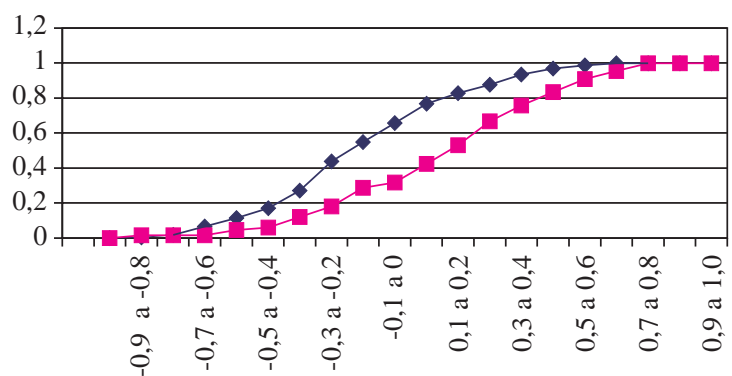

A.4. Pruebas de dominancia estocástica de primer orden para determinar la correlación del crecimiento del PIB (1985-2000)

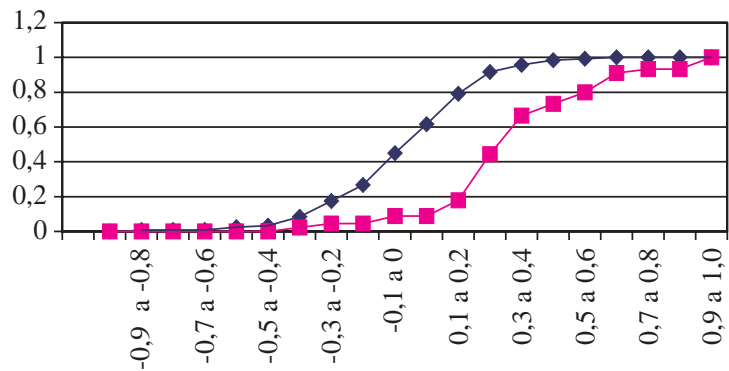


A.5. Pruebas de predominio estocástico de primer orden para determinar la correlación de las tasas de interés real de corto plazo (1985-2000)

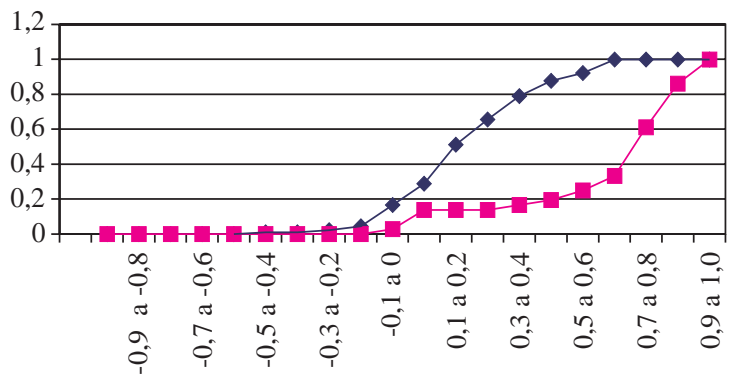

A.7. Pruebas de dominancia estocástica de primer orden para determinar la correlación de las fluctuaciones de la bolsa de valores (IFC. G. COMP: 1990-2002)

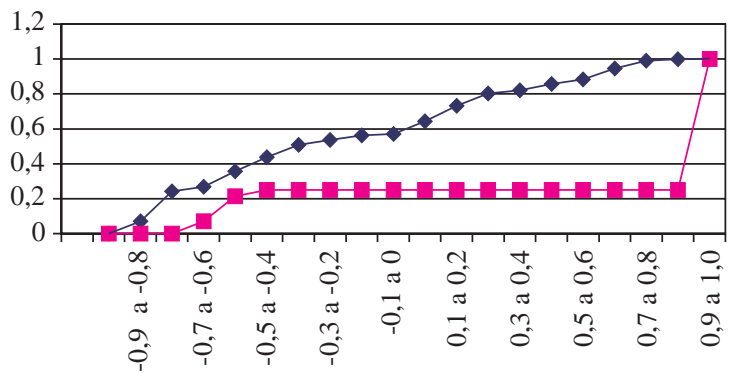

A.6. Pruebas de dominancia estocástica de primer orden para determinar la correlación de las fluctuaciones de la bolsa de valores (IFCI-COMP: 1990-2002)

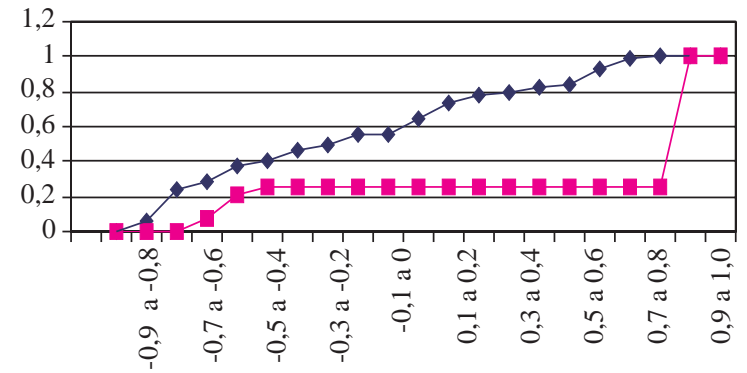

A.8. Pruebas de dominancia estocástica de primer orden para determinar la correlación de las fluctuaciones del mercado de bonos (GBI-EMBI: 1991-2002)

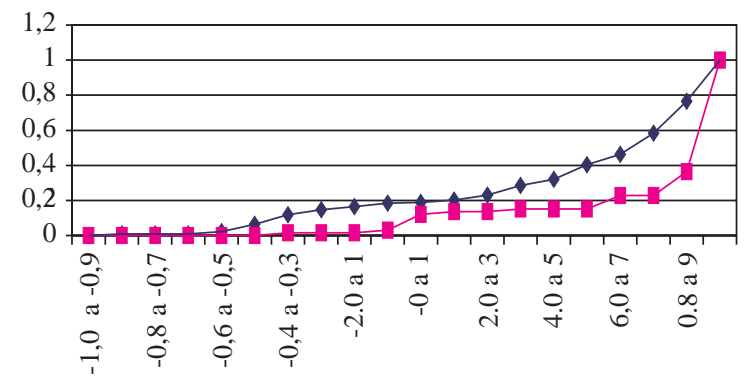

Fuente: Análisis hecho para este estudio.

a Des./en des. $=$ desarrollados/en desarrollo. 


\section{Cálculo de las pérdidas no esperadas}

Dado que la calidad de la cartera crediticia de los bancos puede cambiar en cualquier momento, es necesario calcular frecuentemente las pérdidas esperadas que dicha cartera podría experimentar bajo diferentes situaciones de riesgo. En vista de que dichos cambios en la calidad crediticia son continuos, lo más probable es que el cálculo de las reservas preventivas sea diferente según el período de referencia. La diferencia entre las pérdidas esperadas que se calculan para distintos períodos (debido a los cambios de la calidad crediticia) da lugar a que los bancos puedan sufrir pérdidas, las que en casos extremos podrían llegar a menoscabar su capital. Esta clase de pérdidas se denominan "pérdidas no esperadas" y su cálculo constituye el objeto del presente apéndice.

Las pérdidas no esperadas se generan debido a los cambios en la calidad de los créditos que constituyen una cartera. Para construir un modelo de estas variaciones en la calidad de los créditos, aplicamos un método basado en la teoría de cartera.

Este método ${ }^{6}$ ha sido ampliamente documentado y aplicado en diversos estudios financieros. Según éste, los inversionistas forman su cartera de inversiones prestando especial atención a que exista una relación óptima entre el riesgo y el rendimiento. Para ello, los modeladores de riesgo crediticio han desarrollado técnicas de evaluación de riesgo que toman en cuenta los efectos que puede tener la diversificación de la cartera. Si bien es cierto que estos métodos pueden perfeccionarse, estamos convencidos de que el efecto de la diversificación de la cartera puede y debe formar parte de la evaluación de riesgo con fines regulatorios. ${ }^{7}$ Como sostuvimos en el cuerpo de este trabajo y en documentos anteriores, el hecho de que el marco regulatorio propuesto castigue solamente a quienes asumen un riesgo elevado y no estimule la diversificación de la cartera se traducirá en resultados económicos negativos.

En el presente apéndice presentamos una variante de la metodología CreditMetrics que se ha utilizado para simular las pérdidas no esperadas de los créditos de las carteras examinadas. ${ }^{8}$ En J.P. Morgan (1997) se ha descrito el modelo CreditMetrics como una visión cabal de una cartera de créditos, pues considera las correlaciones entre los créditos que la integran, lo cual determina el costo de la concentración excesiva y las ventajas de la diversificación. El propósito de este apéndice es dar a conocer las modificaciones introducidas en la metodología a fin de hacer posible su apli-

\footnotetext{
${ }^{6}$ Véase Markowitz (1959).

${ }^{7}$ En esta oportunidad, no nos proponemos examinar las mejoras que podrían introducirse en cada método.

${ }^{8}$ Elegimos este modelo debido a la facilidad para construirlo y a la disponibilidad de datos. No pretendemos pronunciarnos en favor de una técnica específica para construir modelos de los riesgos del crédito.
}

cación. ${ }^{9}$ A continuación nos referimos a la versión modificada del CreditMetrics, que hemos denominado "modelo exhaustivo del riesgo del crédito" (Full Credit Risk Model, FCRM).

\section{El modelo exhaustivo del riesgo del crédito}

En diversos estudios empíricos se sostiene que los incumplimientos crediticios están correlacionados. En este trabajo se demuestra que también es posible diversificar el riesgo crediticio. Para calcular la diversificación de la cartera habría que estimar las probabilidades de que cambie conjuntamente la calidad crediticia de cada uno de los créditos que conforman dicha cartera y de que éstos se trasladen desde su categoría crediticia (calidad crediticia) actual a cada una de las categorías crediticias posibles. Para ello necesitaríamos conocer una serie de tablas de probabilidad conjunta equivalente al número de pares de créditos que conforman una cartera. Sin embargo, este objetivo es inalcanzable por la falta de información confiable, la cantidad de datos que sería preciso estimar y la complejidad de construir dicha serie.

La metodología CreditMetrics se basa en dos elementos principales: el método de Merton para construir modelos de las variaciones en la calidad del crédito, y un método indirecto para construir modelos de correlaciones entre los créditos que conforman una cartera.

Finalmente, una vez construida una matriz de correlaciones entre los acreedores, esta metodología simula las pérdidas no esperadas de la cartera.

\section{a) El método de Merton para construir modelos de las variaciones en la calidad del crédito}

El método de Merton supone que el capital propio de una empresa puede concebirse como una opción de compra sobre sus activos con un precio de ejercicio (strike price) igual al valor contable de sus deudas (Merton, 1974). Tras este supuesto está la idea de que, dado que las acciones tienen responsabilidad limitada, los accionistas tienen el derecho, pero no la obligación, de pagar a los tenedores de deuda y hacerse cargo de los restantes activos de la firma. El método implica que la calificación crediticia de un deudor guarda relación con la diferencia entre el valor de mercado de sus activos y su deuda.

De acuerdo con este método, el cambio en el valor de los activos de una empresa determinada se relaciona con el cambio en su calidad crediticia. En consecuencia, para calcular la distribución de las probabilidades de variación de la calidad crediticia, se puede utilizar la distribución de la rentabilidad de los activos de la empresa. Para generalizar el uso de este modelo se debe incluir, además de la condición de

\footnotetext{
${ }^{9}$ Véase una exposición detallada del tema en J.P. Morgan, 1997.
} 
incumplimiento, las posibles distintas calidades que un crédito pueda tener.

La matriz de transición es la variable que resume las probabilidades de transición de una calidad crediticia a otra. Una vez conocidas las probabilidades de transición entre diferentes categorías crediticias, y teniendo en cuenta el modelo de Merton, se puede obtener el valor de mercado de los activos que representan los valores límite entre las distintas categorías, como se aprecia en el gráfico B.1. Estos valores límite cumplen con la condición de que si la variación del valor de mercado del activo $(r)$ es lo suficientemente negativa (es decir, inferior a $Z E$ ) el préstamo cae en incumplimiento; si $Z E<r<Z D$, el crédito pasa a la categoría $D, \mathrm{y}$ así sucesivamente.

Teniendo en cuenta la matriz de transición empírica, se puede calcular la probabilidad de que estos cambios ocurran de la manera que se indica a continuación (para un crédito clasificado inicialmente como $X$ ):

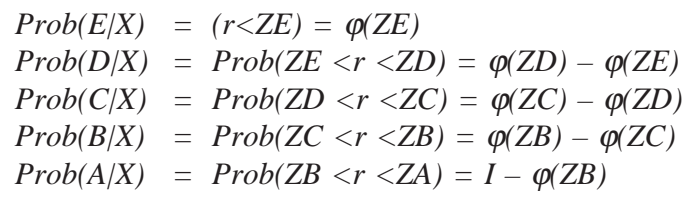
ción normal.

Desde este punto de vista, se puede calcular la matriz de correlación de las variaciones en la calidad crediticia entre deudores, a partir de un modelo que explique las fluctuaciones del valor de los activos de los deudores.

La aplicación de este método presenta varios problemas prácticos, entre los cuales el principal es el manejo de matrices de correlación muy grandes. Además, no se pueden obtener las variaciones del valor de mercado de los ac-

GRÁFICO B.1

\section{Distribución de la rentabilidad de los activos}

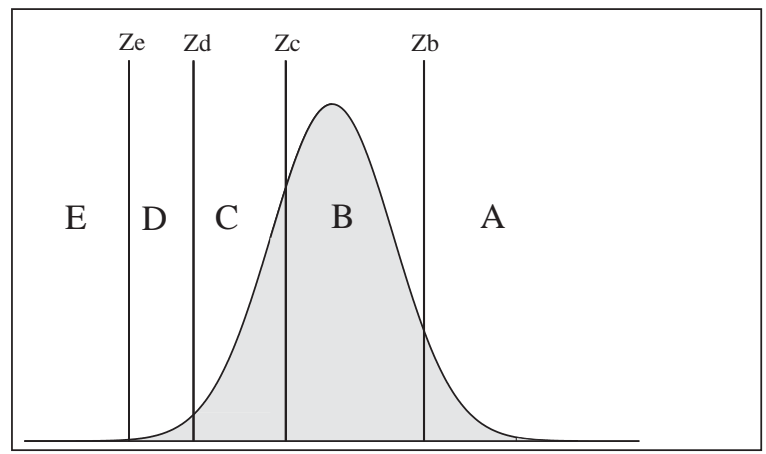

Fuente: Análisis hecho para este estudio. tivos de cada uno de los deudores, ya que para ello sería necesario disponer de información específica sobre la estructura financiera interna de cada uno de ellos. Estos dos inconvenientes impiden aplicar una matriz de correlación ideal, de manera que para introducir el efecto de la diversificación de la cartera, aplicaremos un método indirecto, pero más manejable.

\section{b) Método indirecto para construir modelos de las corre- laciones entre los créditos que conforman una cartera}

A partir de la aplicación del método de Merton, J.P. Morgan (1997) hace una distinción a priori entre los factores que determinan las variaciones del valor de los activos de los deudores. Esta distinción proviene de dos componentes fundamentales: el componente de mercado y el componente idiosincrásico. Por definición, este último no se correlaciona con nada, ya que se refiere a factores propios del deudor. En cambio, el componente de mercado cuenta con todos los elementos que permiten la diversificación de la cartera.

$$
r_{\text {total }}=W_{M} r_{\text {mercado }}+W_{\mathrm{I}} r_{\text {Idiosincrásico }}
$$

donde:

$W_{M} \quad=$ porcentaje de la rentabilidad que corresponde al elemento de mercado. ${ }^{10}$

$\mathrm{r}_{\text {mercado }}=$ componente de mercado de la rentabilidad.

$W_{\text {I }} \quad=$ porcentaje de la rentabilidad que corresponde al elemento idiosincrásico. ${ }^{11}$

$I_{\text {idiosincrásico }}=$ componente idiosincrásico de la rentabilidad.

En cambio, el componente de mercado de la rentabilidad se define como:

$$
r_{\text {Mercado }}=H_{A} r_{\text {PIB país }}+\left(1-H_{A}\right) r_{\text {PIB actividad económica }}
$$

donde:

$H_{A} \quad=$ porcentaje del componente de mercado en el PIB del país deudor, parámetro que se calcula mediante el índice de Herfindahl.

$r_{P I B \text { país }}=$ rentabilidad en el PIB del país deudor.

$\left(1-H_{A}\right)=$ porcentaje del componente de mercado en el PIB del país deudor.

$r_{\text {PIB actividad económica }}=$ rentabilidad en el PIB de la actividad económica del deudor.

${ }^{10}$ En J.P.Morgan (1997) se explica cómo calcular estas ponderaciones. Tras las aplicaciones empíricas, se demuestra que un valor aceptable para $\mathrm{W}$ es $70 \%$, valor que adoptamos para nuestro estudio.

${ }^{11}$ La ponderación del elemento idiosincrásico se obtiene mediante la siguiente ecuación:

$$
w_{I}=\sqrt{1-w^{2}{ }_{M}}
$$

El objetivo de esta ecuación es ser coherente con la variación del valor de mercado de la rentabilidad estandarizada de los activos. 
El componente de mercado de la rentabilidad se divide entre la actividad económica y la región geográfica. Cabe preguntarse qué es más importante para el deudor, su actividad económica o el país en que ésta se realiza. Dado que el porcentaje de participación de estos factores de mercado en el riesgo sistémico del deudor es exógeno al modelo, se aplicó una metodología para resolver este problema de la manera más objetiva posible (Segoviano, 1998).

El método se basó en que mientras más variada sea la actividad económica de un país, menores serán las consecuencias que tendrá un cambio repentino de su producción en el valor de los activos de un deudor en dicho país. Dentro de este marco, es posible deducir que en los países de escasa actividad económica, en que por lo tanto las actividades económicas están muy concentradas, el factor más importante para el valor de los activos del deudor será su ubicación geográfica. Por esta razón, se intuye que, de producirse una perturbación económica en el país, es posible que ella haga disminuir el valor de los activos del deudor, puesto que es muy probable que éste pertenezca al rubro de las actividades económicas afectadas.

De acuerdo con este razonamiento, calculamos un índice de Herfindahl, aplicando la siguiente fórmula para cada grupo de países:

$$
H_{A} \sum_{i=1}^{n}\left(\frac{X_{A i}}{\sum_{j-1}^{n} X_{A j}}\right)^{2}
$$

donde:

$X_{A i}=$ valor de la participación de la actividad económica $i$ en el grupo de países $A .^{12}$

Una vez considerados todos los elementos que constituyen el componente de mercado de la rentabilidad de los activos, se debe calcular la correlación entre los deudores que conforman una cartera de préstamos.

Dada una pareja de deudores, $X$ e $Y$, que se dedican a las actividades industriales, $B$ y $V$, en los grupos de países A y E, su rentabilidad se expresa de la siguiente manera:

$$
r_{X}=w_{I X} r_{I X}+w_{M X} H_{A} r_{A}+w_{M X}\left(1-H_{A}\right) r_{B}
$$

El problema que plantea el cálculo de la correlación entre cada pareja de acreedores de la cartera se resume de la siguiente manera:

$$
\rho_{X Y}=w_{M X} H_{A} w_{M Y} H_{E} \rho_{A E}+w_{M X}\left(1-H_{A}\right) w_{M Y}\left(1-H_{E}\right) \rho_{B V}{ }^{13}
$$

\footnotetext{
${ }^{12}$ Mientras mayor sea el índice de Herfindahl de un grupo determinado de países, menor será la diversificación de la actividad económica. En consecuencia, el porcentaje del componente de mercado explicado por el PIB del país deudor cobra una mayor importancia. 13 Debido a que se parte de la base de que las correlaciones entre los componentes idiosincrásicos y los geográficos, entre los com-
}

donde:

$\rho_{A E}=$ correlación entre los distintos grupos de países. ${ }^{14}$

$\rho_{B V}=$ correlación entre las distintas actividades económicas. ${ }^{15}$

Esta ecuación se resuelve para cada pareja de deudores que conforman la cartera. Los resultados del cálculo de la ecuación se reúnen en una matriz cuadrada $(n \times n)$, donde $n$ es el número de acreedores de la cartera. La matriz se denomina matriz de correlación entre los acreedores y es única para cada cartera. Esta matriz es una variable extremadamente importante para la simulación de pérdidas no esperadas, puesto que incorpora los elementos necesarios para cuantificar la concentración/diversificación de la cartera.

Con estos elementos, en el próximo apartado presentamos la forma en que se simulan los escenarios de calidad de la cartera. A partir de éstos, se hace una estimación de la distribución de las pérdidas, de la cual pueden obtenerse las pérdidas no esperadas.

\section{c) Simulación de escenarios de calidad de la cartera de préstamos}

Al combinar la matriz de transición con la matriz de correlación entre los acreedores, simulamos escenarios de calidad, a partir de los cuales se obtiene la distribución de las pérdidas de la cartera crediticia.

Como se señaló anteriormente, la matriz de transición indica las probabilidades de cambio en la calidad crediticia que podrían afectar a los acreedores de una categoría determinada. Además, se tiene en cuenta la correlación de las variaciones en la calidad de los acreedores. Es decir, cuando se produzcan perturbaciones económicas, los acreedores con características similares tenderán a migrar juntos a categorías de crédito similares. En cambio, si sus características son diferentes, generalmente migrarán por separado a categorías de crédito diferentes. Ello implica que las carteras concentradas en créditos con características semejantes mostrarán una mayor tendencia a sufrir más pérdidas no esperadas, debido a que no diversificarán los posibles riesgos económicos.

Por otra parte, programamos un algoritmo para calcular diez mil escenarios de calidad posibles para cada una de las $(n \times n)$ parejas de acreedores que conforman la cartera. Cada escenario de calidad muestra un cambio en el valor de

ponentes idiosincrásicos y los de la actividad económica, y entre los componentes de la actividad económica y los geográficos son iguales a cero.

${ }^{14}$ Estas correlaciones se calcularon entre los márgenes de los créditos sindicados correspondientes a cada grupo de países. Consideramos que estos márgenes representan el grado de riesgo del sistema financiero de cada grupo de países.

${ }^{15}$ Estas correlaciones se calcularon entre los índices correspondientes a cada una de las actividades económicas consideradas en el estudio. Cada índice de actividad económica se construyó tomando en cuenta la proporción de la actividad económica en el PIB de un país representativo de cada grupo de países de la muestra. 
mercado de los activos de los acreedores de la cartera. Este proceso se repitió diez mil veces. Los cambios de categoría de los integrantes de la cartera permiten generar un volumen de pérdidas o utilidades que representan la distribución de las pérdidas de la cartera.

Para generar estos escenarios, se llevó a cabo el siguiente procedimiento:

i) Generación de números aleatorios uniformes.

ii) Transformación de estos números aleatorios en números aleatorios estándares normales.

iii) Transformación de los números aleatorios estándares normales en números aleatorios múltiples normales con variación igual a la matriz de correlación entre los acreedores.

Como se partió de la base de que el proceso que genera las variaciones en los activos sigue una distribución normal, utilizamos una distribución aleatoria múltiple normal a fin de generar migraciones conjuntas de calidad, en que los créditos con un alto grado de correlación tienden a trasladarse conjuntamente.

\section{d) Las pérdidas no esperadas}

Una vez simulados los escenarios de calidad de la cartera de préstamos, se pueden calcular las pérdidas y/o ganancias que genera la diferencia entre la categoría inicial y final del crédito. Las pérdidas o ganancias obtenidas del proceso de simulación se utilizaron para construir un histograma, en el que se resume la distribución de las pérdidas de la cartera.
GRAFICO B.

\section{Distribución de las pérdidas de la cartera crediticia}

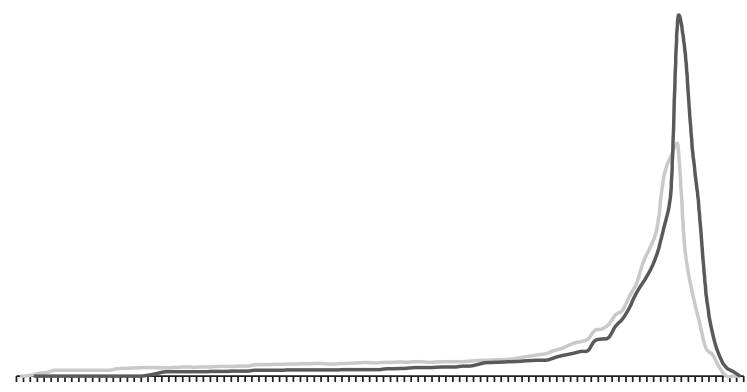

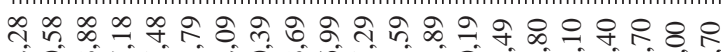

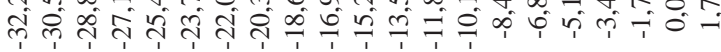

\section{- Desarrollados/emergentes Desarrollados}

Fuente: Análisis hecho para este estudio.

Para obtener la distribución de las pérdidas, es necesario ordenar las pérdidas no esperadas simuladas. A partir de esta distribución, se determina un modelo de cálculo del valor en riesgo, del cual se obtiene el monto de las pérdidas no esperadas de la cartera. Al dividir las pérdidas no esperadas por el valor total de la cartera, se obtiene el porcentaje, con una probabilidad dada, que podría perderse en un caso extremo. Por lo tanto, los requerimientos de capital deberían ser los necesarios para que pudiesen absorber esas pérdidas.
Acharya, V., I. Hasan y A. Saunders (2002): Should Banks Be Diversified? Evidence from Individual Bank Loan Portfolios, BIS Documento de Trabajo No 118, Basilea, Banco de Pagos Internacionales (BPI), septiembre.

Comité de Basilea de Supervisión Bancaria (2002): Basel Committee reaches agreement on New Capital Accord issues, http:// www.bis.org/press/p020710.htm.

Davis, E.P. (1999): Russia/LTCM and market liquidity risk, The Financial Regulator, vol. 4/2, Londres.

Griffith-Jones, S. (2002): Capital Flows to Developing Countries: Does the Emperor Have Clothes?, Sussex, Institute of Development Studies.

Griffith-Jones, S. y S. Spratt (2001): Will the proposed new Basel Capital Accord have a net negative effect on developing countries?, Brighton, Institute of Development Studies.

Griffith-Jones, S., S. Spratt y M. Segoviano (2002): Basel II and developing countries, The Financial Regulator, vol. 7, $\mathrm{N}^{\circ}$ 2, septiembre.
J.P. Morgan (1997), CreditMetrics Technical Document, Nueva York. Kaminsky, G. y C. Reinhart (2002), The Center and the Periphery: The Globalization of Financial Turmoil, Washington, D.C., George Washington University, versión preliminar.

Kaminsky, G., C. Reinhart y C. Vegh (2002): Two Hundred Years of Contagion, en prensa.

Markowitz, Harry (1959): Portfolio Selection: Efficient Diversification of Investments, Nueva York, John Wiley and Sons.

Merton, Robert C. (1974): On the pricing of corporate debt: The risk structure of interest rates, Journal of Finance, vol. 29.

Persaud, A. (2001): Sending the Herd off the Cliff-Edge: The Disturbing Interaction Between Herding and Market-Sensitive Risk Management Practices, State Street Bank, marzo.

Segoviano, M. (1998): Basel II vs. CreditMetrics, Londres, London School of Economics.

Zigrand, J.P. y J. Danielsson (2001): What Happens When You Regulate Risk? Evidence from a Simple Equilibrium Model, FMG Documento de Trabajo No 393 , Londres. 